\title{
Effects of local anesthetics on the respiratory burst of cord blood neutrophils in vitro
}

\author{
Hanna Billert' ${ }^{1}$ Katarzyna Czerniak', Ewa Bednarek and Karolina Kulińska'
}

BACKGROUND: Whether local anesthetics exert anti-inflammatory effects in fetal and newborn systemic neutrophils is unclear. The aim of the present study was to assess the effects of bupivacaine and lidocaine on the respiratory burst of cord blood neutrophils in vitro compared with adult cells.

METHODS: Whole cord blood $(n=12)$ and control adult blood samples $(n=7)$ were incubated with bupivacaine $(0.0005$, $0.005,0.05,1 \mathrm{mmol} / \mathrm{l})$ and lidocaine $(0.002,0.02,0.2,4 \mathrm{mmol} / \mathrm{l})$ for 1 and $4 \mathrm{~h}$. The production of reactive oxygen species (ROS) by unstimulated neutrophils and the phorbol myristate acetate-induced oxidative burst were assessed by flow cytometry. A subset of neutrophils showing high fluorescence intensity (rho+) was analyzed separately.

RESULTS: After $1 \mathrm{~h}$ incubation, local anesthetics decreased the respiratory burst in whole cord blood and adult neutrophils in a similar manner. At the clinically relevant concentration of $0.0005 \mathrm{mmol} / \mathrm{l}$, bupivacaine was active, but its effect in cord blood cells could not be detected after $4 \mathrm{~h}$. The cord blood rho+ cell subset was unresponsive to the inhibitory action of bupivacaine. In rho+ neutrophils, basal ROS production was stimulated by lidocaine at the lowest concentration tested.

CONCLUSION: Bupivacaine and lidocaine can decrease the respiratory burst in neutrophils of term newborns.

$\mathbf{T}$ he question of the effects of local anesthetics (LAs) on fetal neutrophils arises in the clinical context of the broad application of neuraxial blockade in obstetrics. Epidural technics are the gold standard for labor intrapartum analgesia and are administered to parturients on request. By applying higher doses of LAs through the existing epidural catheter, surgical anesthesia for cesarean delivery may be easily obtained. The fetal and neonatal implications of epidural techniques are mostly considered to be indirect and dependent on modulation of maternal physiology, and are associated with a higher risk of neonatal pyrexia and sepsis evaluation, but not neonatal infection (1-3). The reasons for these findings are not sufficiently clear. However, direct fetal and neonatal effects of LAs as a result of placental drug transfer should not be entirely excluded. LAs administered epidurally enter the maternal systemic circulation and, after crossing the placental barrier, can reach the fetal circulation, with levels approximately one-third to half those seen in the maternal circulation. Furthermore, because of ion trapping and slower elimination, LAs may persist for longer in fetal and neonatal than maternal blood (4). LAs in the fetal systemic circulation have been reported to abolish an offspring's cardiovascular adaptive reaction to asphyxia (5) and have been suggested to affect blood cells. Specifically, bupivacaine has been shown to modify plasma membrane properties of fetal erythrocytes (6). In other studies, lidocaine, a prototypical amino-amide agent administered epidurally for cesarean sections, was suggested to be responsible for the inhibition of natural killer cells and neutrophil activities in newborns $(7,8)$. Recent research supports decreased chemotactic response in infants delivered by cesarean section under epidural anesthesia as compared to single shot spinal technique with negligible penetration of LAs to fetal circulation (9). It seems therefore that LAs themselves may be important for inflammatory neonatal implications of neuraxial blockades.

The anti-inflammatory actions of LAs, documented in various experimental and clinical settings, are of considerable interest because of potential clinical implications (10-12). LAs are able to modulate humoral and cellular aspects of the inflammatory response (i.e., they can suppress various functions of neutrophils, which are essential players in innate immunity). Generation of reactive oxygen species (ROS) and the respiratory burst due to activation of nicotinamide adenine dinucleotide phosphate-oxidase (NOX), critical for antimicrobial activity, cell signaling, and life span, are inhibited by LAs through interference with $G_{\alpha q}$ protein signaling (10). Whether LAs exert anti-inflammatory effects in fetal systemic neutrophils is unclear.

Neutrophils are especially vital for infection control at the time of transition to extrauterine life when mechanisms of specific immunity are underdeveloped. Phenotypically and functionally, fetal neutrophils differ significantly from adult neutrophils (13). Data are inconsistent regarding the capacity of oxidative phenomena in fetal and neonatal cells (13-15). Nevertheless, the physiology of cell systems responsible for 
ROS generation is substantially different in fetal and neonatal cells and may be less effective under septic conditions $(13,14)$. Because of these discrepancies, an altered susceptibility toward drug effects on oxidative metabolism cannot be excluded. Because ROS production is critical for neutrophil performance, the effects of LAs on this process in the fetus need to be elucidated.

The aim of the present study was to assess the effects of the LAs, bupivacaine and lidocaine, on the respiratory burst of cord blood polymorphonuclear neutrophils in vitro compared with that in adult cells. Our hypothesis was that LAs would suppress the respiratory burst to a significantly greater extent in cord blood neutrophils than in adult neutrophils.

\section{RESULTS}

\section{Obstetric and Infant Characteristics}

Obstetric and infant data are presented in Table 1.

Table 1. Obstetric and infant data

\begin{tabular}{lc}
\hline Parity: primipara/multipara & $6 / 6$ \\
\hline Gestational age (weeks) & $39(38-40)^{\mathrm{a}}$ \\
Duration of membrane rupture (min) & $305(185,425)$ \\
Duration of the first stage (min) & $343(239,446)$ \\
Duration of delivery (min) & $18(10-40)^{\mathrm{a}}$ \\
Meperidine analgesia (yes/no) & $8 / 4$ \\
Total dose of meperidine $(\mathrm{mg})$ & $100(50-125)^{\mathrm{a}}$ \\
Maternal temperature $\left({ }^{\circ} \mathrm{C}\right)$ & $36.6(36.5,36.8)$ \\
Infants gender: male/female & $6 / 6$ \\
Infant body mass (g) & $3,369(3,031,3,708)$ \\
Placental mass (g) & $533(460,605)$ \\
Apgar score & $10(10)^{\mathrm{a}}$ \\
$\mathrm{pH}_{\mathrm{a}}$ & $7.22(7.17,7.28)$ \\
$P_{\mathrm{a}} \mathrm{O}_{2}$ (mmHg) & $25.0(13.9,36.1)$ \\
$P_{\mathrm{a}} \mathrm{CO}_{2}$ (mmHg) & $57.0(50.1,63.9)$ \\
$\mathrm{BEa}$ & $-5.5(-8.1,-2.9)$ \\
\hline Normally distributed data are presented as numbers or as mean values \\
$(95 \%$ confidence intervals); non-normally distributed data are given as median values \\
(interquartile range). \\
$\mathrm{BEa}_{\text {, arterial cord blood base excess; } \mathrm{pH}_{\mathrm{a}^{\prime}} \text { arterial cord blood pH. }}$
\end{tabular}

\section{Neutrophil ROS Production in Control Cord and Adult Blood}

Neutrophil ROS generation in control samples (without the addition of LAs) was quantitatively comparable between fetal and adult cells across the entire neutrophil population. However, in the subset of neutrophils showing high fluorescence intensity (rho+), unstimulated fetal neutrophils exhibited much higher basal oxidative activity than adult control neutrophils after $1 \mathrm{~h}$ incubation (Table 2).

The proportion of phorbol myristate acetate (PMA)stimulated rho+ neutrophils in cord blood was lower than in adult control samples (Table 3 ).

A stimulation index (mean fluorescence intensity (MFI) of stimulated cells/MFI of unstimulated cells) was calculated for the entire neutrophil population at a basal state (without LA). Stimulation index median (interquartile range) values after 1 and $4 \mathrm{~h}$ incubation for cord blood cells were $51(45,94)$ and $40(22,72)$, respectively, and for adult cells $41(22,59)$ and 44 $(13,59)$, respectively (Mann-Whitney $U$-test). No differences between cord blood and adult neutrophils and between both incubation times studied were noted.

\section{Effects of LAs on Neutrophil ROS Production in Cord and Adult Blood}

Whole neutrophil population. At the lowest, clinically relevant, concentration $(0.0005 \mathrm{mmol} / \mathrm{l})$, bupivacaine treatment resulted in a decrease in the PMA-elicited respiratory burst (Figure 1a and Figure 2a-d). This effect was comparable in cord blood and adult neutrophils after $1 \mathrm{~h}$ incubation; however, after $4 \mathrm{~h}$, the effect only persisted in adult cells (Figure $2 \mathrm{c}$ ). At $1 \mathrm{mmol} / \mathrm{l}$, bupivacaine did not inhibit the respiratory burst in either fetal or adult neutrophils after 1 and $4 \mathrm{~h}$ incubation (Figure 2c).

Lidocaine significantly suppressed the respiratory burst in both cord blood and adult neutrophils at the highest concentration tested $(4 \mathrm{mmol} / \mathrm{l})$ after 1 and $4 \mathrm{~h}$ incubation (Figure 2d).

Rho+ neutrophil cell subset. Bupivacaine had no effect in either unstimulated or PMA-stimulated cord blood neutrophils (Figure 1b; Figure 3a-d). In adult cells, bupivacaine decreased the respiratory burst at lower concentrations (Figure 3a,c).

Table 2. Production of reactive oxygen species by neutrophils from cord and adult blood control samples after incubation for 1 or $4 \mathrm{~h}$

\begin{tabular}{|c|c|c|c|c|c|c|c|c|}
\hline & \multicolumn{4}{|c|}{ One hour incubation } & \multicolumn{4}{|c|}{ Four hours incubation } \\
\hline & \multicolumn{2}{|c|}{ Entire sample } & \multicolumn{2}{|c|}{ rho+ subset } & \multicolumn{2}{|c|}{ Entire sample } & \multicolumn{2}{|c|}{ rho+ subset } \\
\hline & Unstimulated & $\begin{array}{c}\text { PMA } \\
\text { stimulated }\end{array}$ & Unstimulated & $\begin{array}{c}\text { PMA } \\
\text { stimulated }\end{array}$ & Unstimulated & $\begin{array}{c}\text { PMA } \\
\text { stimulated }\end{array}$ & Unstimulated & PMA stimulated \\
\hline \multicolumn{9}{|c|}{ ROS production (MFI) } \\
\hline $\begin{array}{l}\text { Cord blood } \\
(n=12)\end{array}$ & $39(29-53)$ & $\begin{array}{c}3,741 \\
(2,372-6,014)\end{array}$ & $\begin{array}{c}1,127^{\mathrm{a}} \\
(867-1,304)\end{array}$ & $\begin{array}{c}3,807 \\
(2,665-6,737)\end{array}$ & $38(28-52)$ & $\begin{array}{c}2,121 \\
(1,405-3,191)\end{array}$ & $\begin{array}{c}1,046 \\
(644-1,252)\end{array}$ & $\begin{array}{c}2,481 \\
(1,655-4,688)\end{array}$ \\
\hline
\end{tabular}

Data are presented as median values with the interquartile range in parentheses.

aSignificant difference between cord and adult cells after $1 \mathrm{~h}$ in unstimulated rho+ neutrophils ( $P=0.036$; Mann-Whitney $U$-test).

$\mathrm{MFI}$, mean fluorescence intensity; rho+, neutrophil cell subset with high-intensity fluorescence; PMA, phorbol myristate acetate. 


\section{Articles $\mid$ Billertetal.}

Table 3. Proportion of neutrophils with high-intensity fluorescence (rho+) in the entire neutrophil population of cord and adult blood after incubation with or without local anesthetics for 1 or $4 \mathrm{~h}$

\begin{tabular}{|c|c|c|c|c|c|}
\hline & \multirow[b]{3}{*}{$\mathrm{LA}(\mathrm{mmol} / \mathrm{l})$} & \multicolumn{4}{|c|}{$\%$ rho+ neutrophils } \\
\hline & & \multicolumn{2}{|c|}{ One hour incubation } & \multicolumn{2}{|c|}{ Four hours incubation } \\
\hline & & Unstimulated & PMA stimulated & Unstimulated & PMA stimulated \\
\hline Control & 0 & $7.1(4.9-11.0)$ & $93.9^{\dagger}(90.0-97.4)$ & $8.0(5.4-11.0)$ & $91.4^{\ddagger}(78.0-94.0)$ \\
\hline \multirow[t]{3}{*}{ Bupivacaine } & 0.0005 & $6.9(4.5-8.3)$ & $93.8(88.5-95.3)$ & $5.6(4.6-9.0)$ & $87.8(81.7-93.5)$ \\
\hline & 0.05 & $6.0(4.7-8.4)$ & $92.2(88.6-94.6)$ & $6.2^{*}(4.7-7.7)$ & $91.7(85.9-95.3)$ \\
\hline & 1 & $4.9(5.6-10.2)$ & $93.3(89.4-95.0)$ & $5.6^{*}(4.2-9.2)$ & $94.1(89.7-96.3)$ \\
\hline \multirow[t]{3}{*}{ Lidocaine } & 0.002 & $5.7(4.1-8.4)$ & $92.4(84.8-93.8)$ & $6.4^{* *}(4.8-8.3)$ & $88.6(76.1-94.0)$ \\
\hline & 0.02 & $6.9(5.6-9.4)$ & $90.6(85.4-95.9)$ & $6.3(4.9-8.9)$ & $92.9(75.9-96.8)$ \\
\hline & 0.2 & $7.4(5.7-10.3)$ & $93.2(83.4-95.3)$ & $6.8(6.5-10.4)$ & $87.4(76.8-92.8)$ \\
\hline \multicolumn{6}{|c|}{ Adult blood neutrophils ( $n=7$ ) } \\
\hline \multirow[t]{4}{*}{ Bupivacaine } & 0.0005 & $3.3(2.3-7.6)$ & $97.0(89.0-98.8)$ & $3.3^{* *}(2.4-6.8)$ & $84.0(80.7-97.4)$ \\
\hline & 0.005 & $3.1(2.5-7.6)$ & $98.1(82.3-99.2)$ & $2.7(2.5-6.6)$ & $94.3(84.8-95.8)$ \\
\hline & 0.05 & $4.5(2.8-11.0)$ & $88.5(84.8-96.2)$ & $3.1(2.7-5.5)$ & $95.3(86.1-97.4)$ \\
\hline & 1 & $2.9 *(2.4-3.3)$ & $99.0(90.8-99.1)$ & $3.0(2.5-3.2)$ & $95.8(94.4-96.4)$ \\
\hline \multirow[t]{4}{*}{ Lidocaine } & 0.002 & $4.0(2.6-5.6)$ & $86.5(80.7-94.7)$ & $2.5^{* *}(2.3-6.1)$ & $89.1(72.1-95.6)$ \\
\hline & 0.02 & $2.8(2.4-7.7)$ & $86.5(82.3-98.9)$ & $3.6(2.3-9.4)$ & $94.7^{*}(81.4-96.3)$ \\
\hline & 0.2 & $3.9(2.9-9.9)$ & $91.9(78.7-98.1)$ & $3.1(2.7-8.1)$ & $88.8(78.3-93.2)$ \\
\hline & 4 & $3.7(2.8-10.7)$ & $72.2^{* *}(57.1-73.3)$ & $3.4(2.8-7.7)$ & $67.2^{*}(57.2-82.0)$ \\
\hline
\end{tabular}

Data are presented as median values with the interquartile range in parentheses.

${ }^{*} P<0.05,{ }^{* *} P<0.01$ compared with control (Friedman and Dunn); ${ }^{+} P=0.047,{ }^{\ddagger} P=0.010$ compared with adult neutrophils (Mann-Whitney $U$-test).

LA, local anesthetic; PMA, phorbol myristate acetate.

Lidocaine increased ROS generation in intact rho+ neutrophils (Figure $3 \mathbf{b}$ ), but decreased the respiratory burst comparably in cord and adult cells at the highest concentration tested after $1 \mathrm{~h}$ incubation. After $4 \mathrm{~h}$ incubation, the effect in cord blood cells was no longer evident (Figure 3d).

Major effects of LAs on neutrophil ROS production in cord and adult blood are summarized in Table 4.

\section{DISCUSSION}

The present study reports for the first time direct effects of LAs on cord blood neutrophil performance regarding ROS generation in an in vitro whole-blood model. The most important findings of the present study are that: (i) bupivacaine, at clinically relevant concentrations, is able to decrease the PMAinduced respiratory burst in cord blood neutrophils in the short-term, but that upregulated rho+ cells are resistant against this inhibitory action; and (ii) in a subset of unstimulated rho+ cord blood neutrophils, lidocaine was able to increase ROS generation at the lowest concentration tested.

The inhibitory effects of bupivacaine observed at the lowest concentration tested in both cord blood and adult neutrophils (in contrast with the effects of lidocaine) underscore cell sensitivity to this agent and correspond to its marked membrane interaction, a feature that contributes to its cardiotoxicity (16).

In isolated adult neutrophils, bupivacaine was reported not to suppress the PMA-stimulated respiratory burst until it was applied at millimolar concentrations (17); some authors have rejected any effect of bupivacaine on neutrophil oxidant production (18). In the whole-blood model, a decrease in neutrophil respiratory burst elicited by microbial phagocytosis following bupivacaine exposure was demonstrated over the micromolar to millimolar concentration range $(12,19)$. In the present study, we found that bupivacaine could affect oxidative phenomena in systemic neutrophils at a clinically relevant concentration. Intriguingly, the inhibitory effect observed at the lowest concentration of bupivacaine was not evident with the highest concentration used, suggesting a nonlinear response. As a mitochondrial decoupler, bupivacaine is able to increase the leak of mitochondrial ROS, which, in human leukocytes, may activate NOX and thus alleviate the inhibitory actions of the drug (20). However, the effect of bupivacaine on neutrophil mitochondria was recently challenged (21). Because we used dihydrorhodamine (DHR) as the fluorescent probe, the contribution of nitric oxide synthesized by neutrophils following 
a

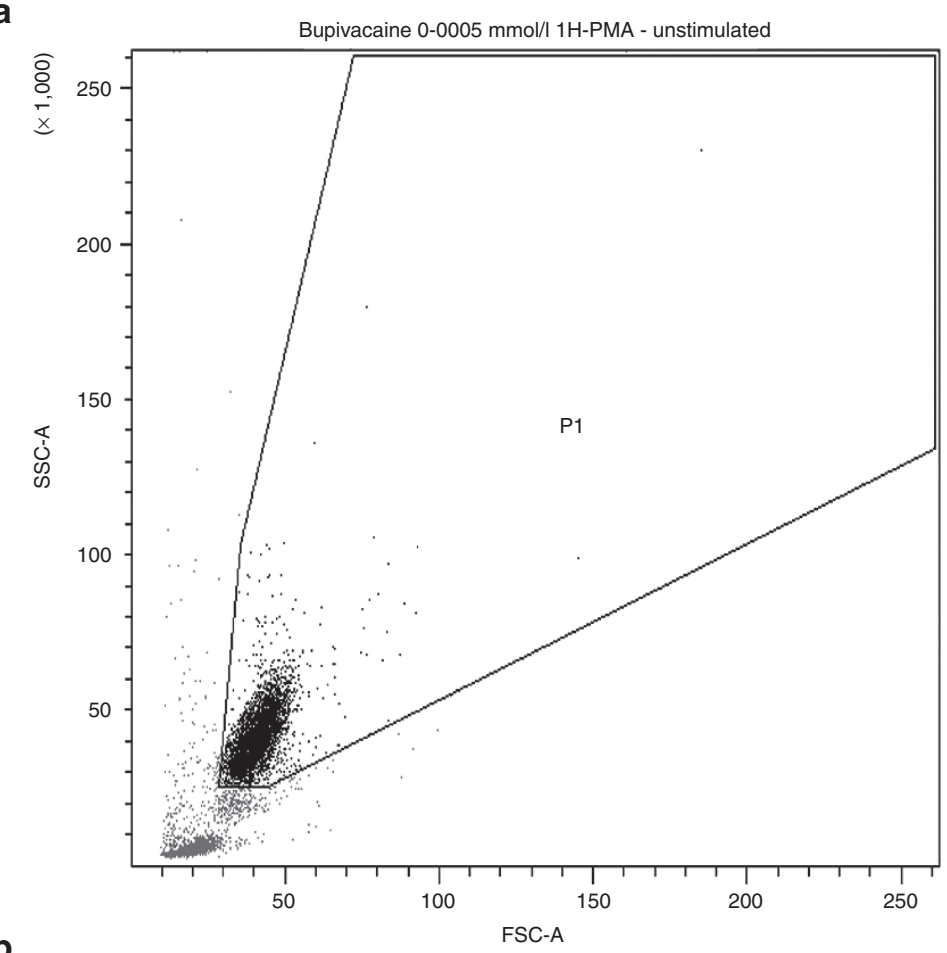

b

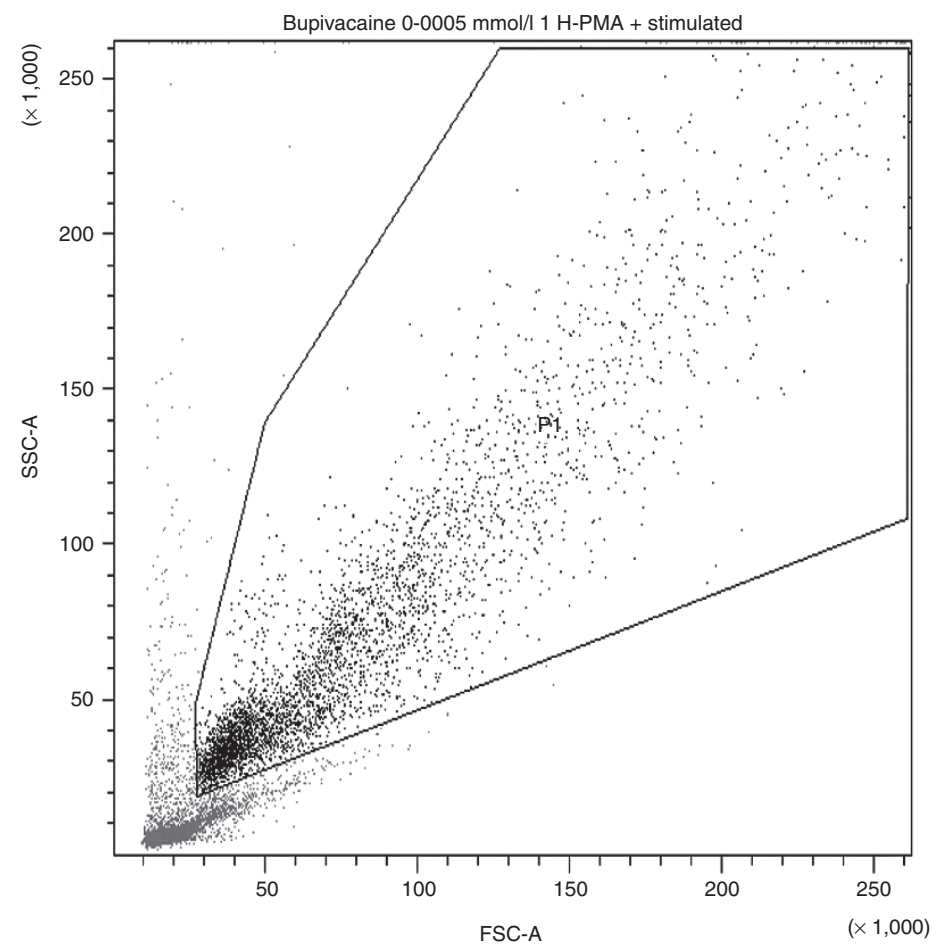

Bupivacaine 0-0005 mmol// 1H-PMA - unstimu
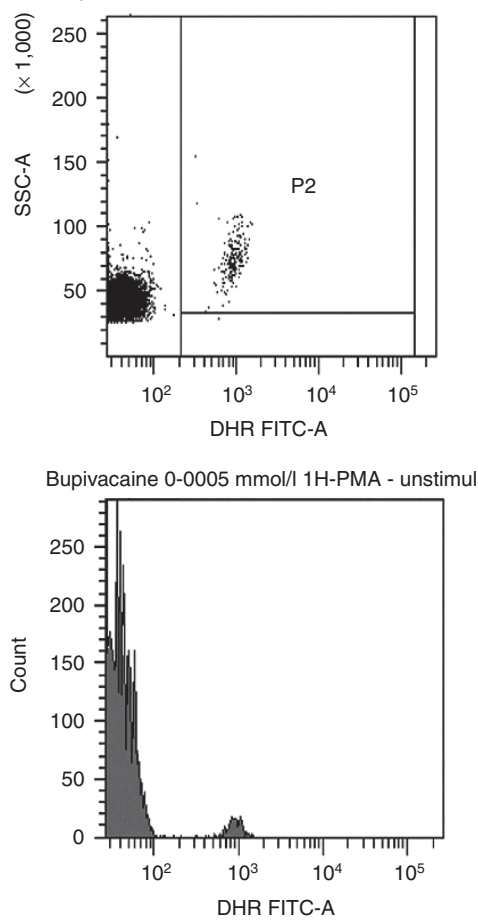

Bupivacaine 0-0005 mmol// 1H-PMA + stimulate
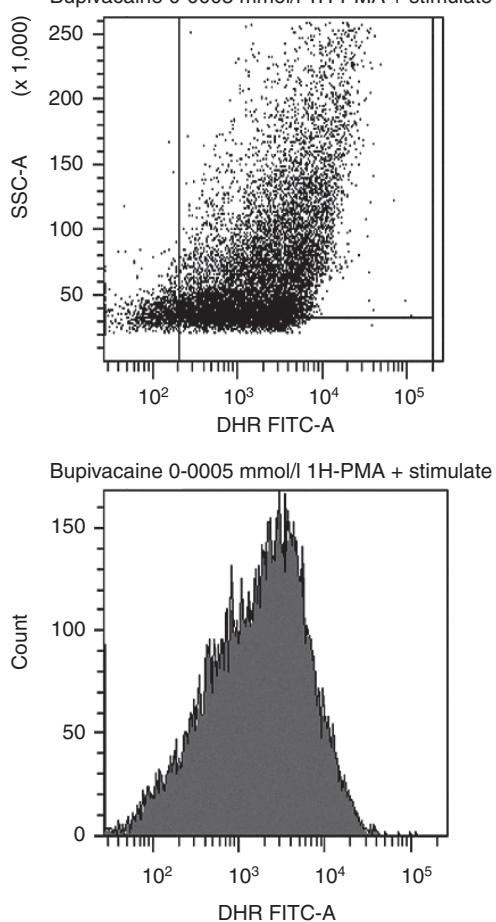

Figure 1. Reactive oxygen species (ROS) generation by neutrophils. Representative cytometric dot-plots for reactive oxygen species generation in (a) unstimulated cord blood neutrophils and (b) after stimulation of the respiratory burst with phorbol myristate acetate. Gate P1 represents the whole neutrophil population, whereas Gate P2 represents the cell subset with high-intensity fluorescence (rho+).

PMA stimulation cannot be excluded: LAs may enhance this effect (22).

Exposure of both cord blood and adult neutrophils to lidocaine resulted in suppression of the respiratory burst only with the highest concentration used, and therefore these actions of lidocaine appear not to be clinically relevant. In isolated neutrophils, an inhibitory effect of lidocaine on PMA-elicited ROS generation has also been reported with high concentrations, as high as double those used in the present study (23). Lidocaine has been reported to suppress the respiratory burst 


\section{Articles | Billert et al.}

a
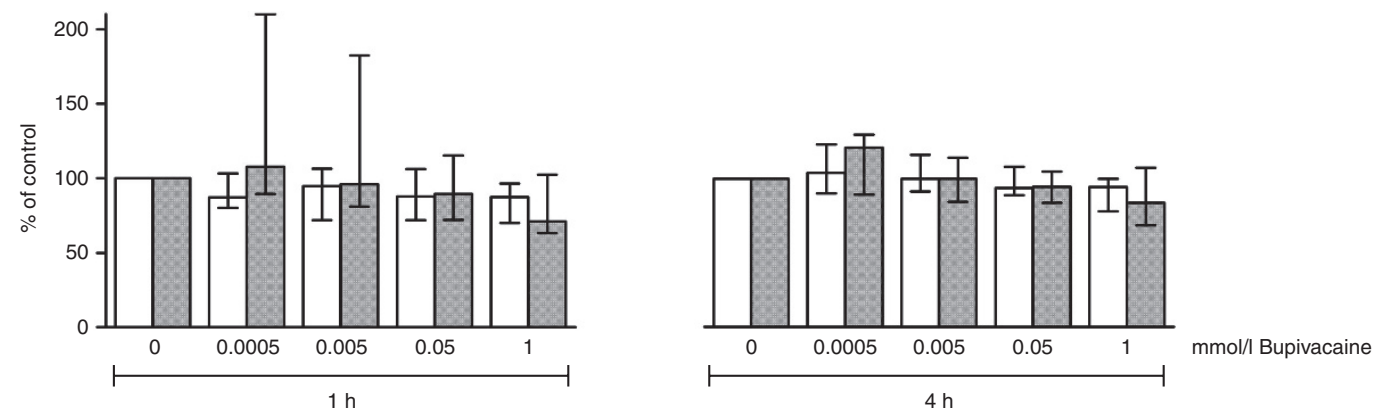

b
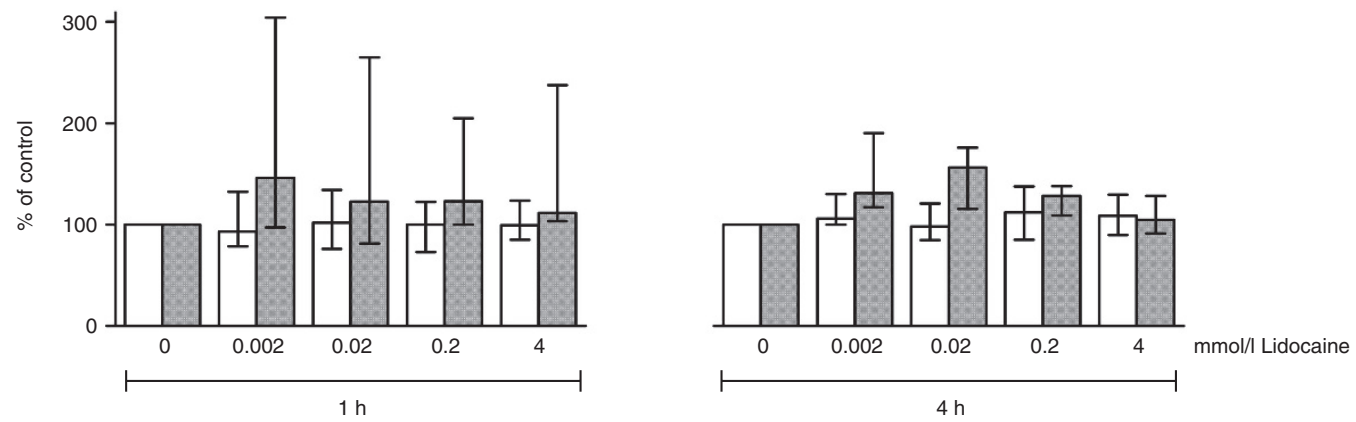

C
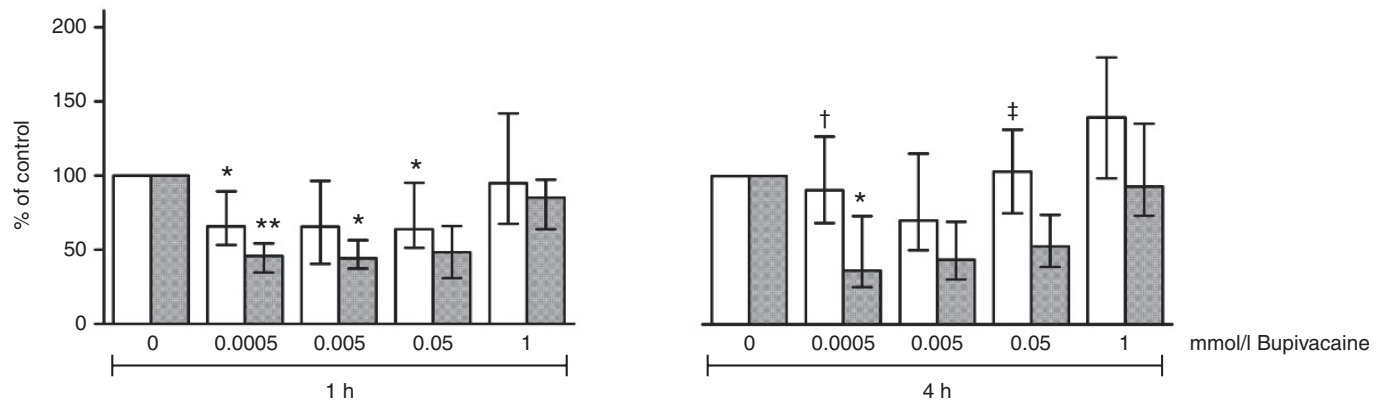

d
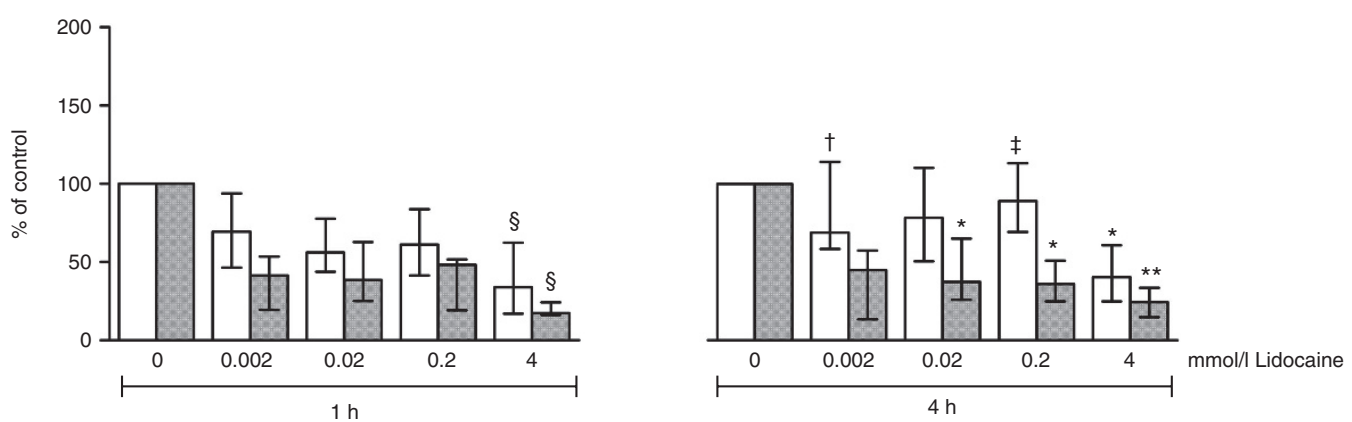

Figure 2. Reactive oxygen species (ROS) generation by neutrophils. Effects of bupivacaine and lidocaine on reactive oxygen species (ROS) production by (a, b) unstimulated neutrophils and $(\mathbf{c}, \mathbf{d})$ after stimulation of the respiratory burst with phorbol myristate acetate (PMA) in the entire neutrophil cell population in cord blood ( $n=12$; white bars) and adult control samples ( $n=7$; gray bars) after 1 and $4 \mathrm{~h}$ incubation. Intracellular ROS generation was assessed by flow cytometry using dihydrorhodamine (DHR) 123 as a fluorescent probe. $14.35 \mu \mathrm{mol} / \mathrm{I} \mathrm{DHR} 123$ was immediately added to whole blood samples after the $5 \mathrm{~min}$ incubation with $0.97 \mu \mathrm{mol} / \mathrm{I}$ PMA or vehicle. Unstimulated and PMA-stimulated cells were analyzed separately. Data (median and interquartile range) are percentage of control values (without an anesthetic). ${ }^{*} P<0.05,{ }^{*} P<0.01, \S P<0.001$ compared with control (Friedman and Dunn). (a, b) Bupivacaine and lidocaine did not affect ROS generation in the entire population of unstimulated cord blood and control adult neutrophils. (c) Bupivacaine at the lowest, clinically relevant concentration $(0.0005 \mathrm{mmol} / \mathrm{l})$ decreased ROS generation in the PMA-stimulated cord blood and adult control samples after $1 \mathrm{~h}$ incubation. After $4 \mathrm{~h}$, the inhibitory effect persisted only in adult cells; differences between cord blood and adult neutrophils: $\uparrow P=0.025, \neq P=0.008$ (Mann-Whitney $U$-test). (d) Lidocaine decreased the PMA-elicited respiratory burst in cord blood and adult blood neutrophils only at the highest concentration applied (4 mmol/l) after both 1 and $4 \mathrm{~h}$ incubation. In adult control samples, inhibitory effects were additionally observed at lower concentrations after $4 \mathrm{~h}$. Differences between cord blood and adult neutrophils: $\uparrow P=$ $0.020, \neq P=0.023$ (Mann-Whitney $U$-test). 
a

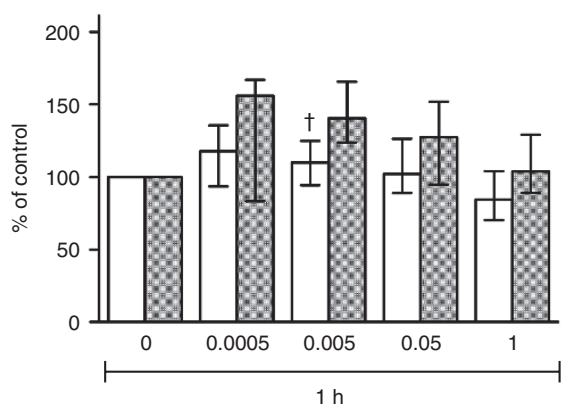

b

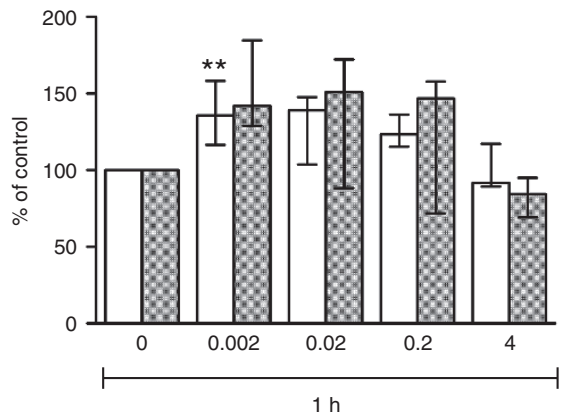

C

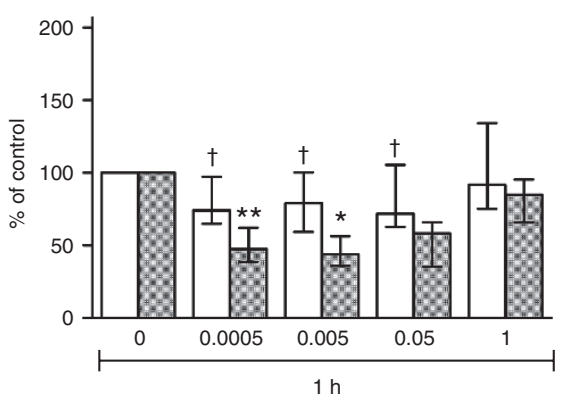

d

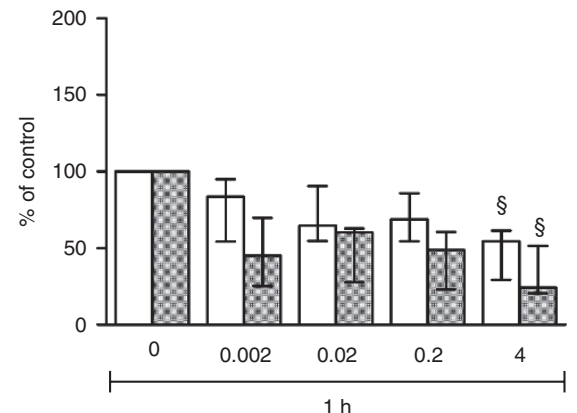

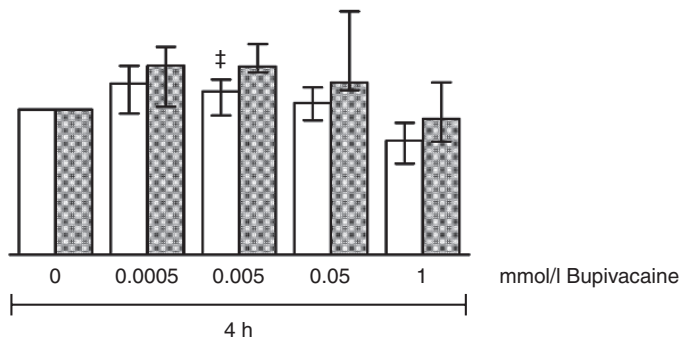
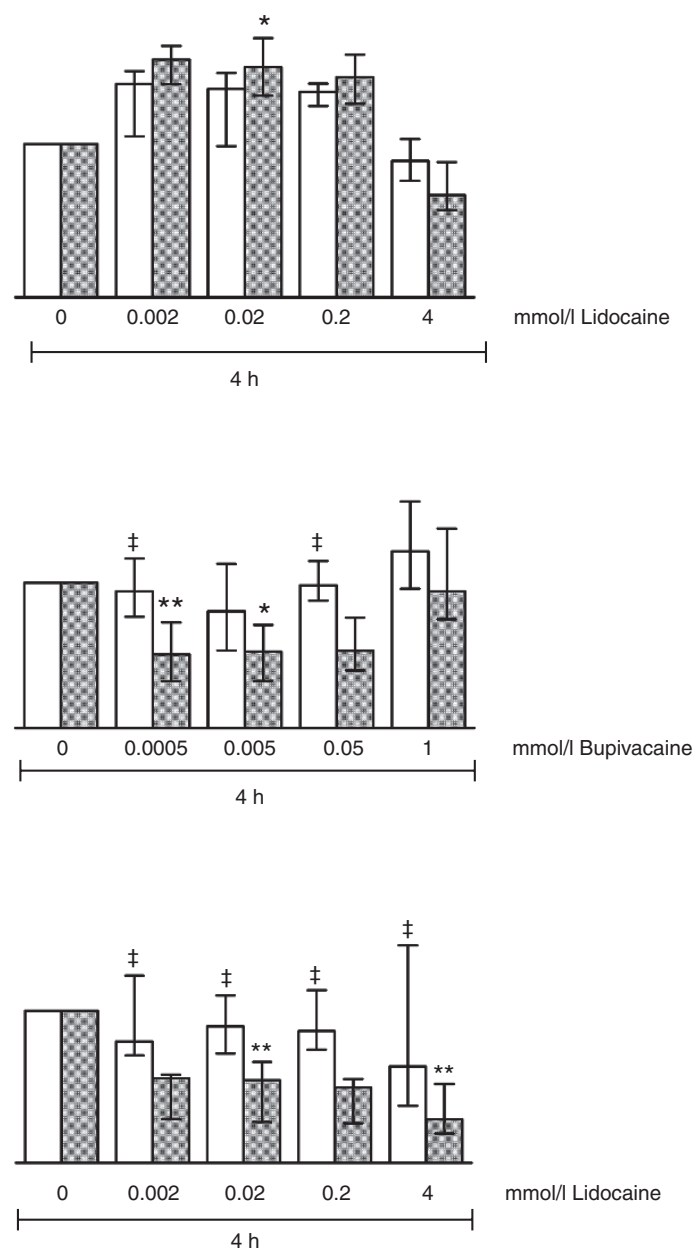

Figure 3. Reactive oxygen species (ROS) generation by neutrophils. Effects of bupivacaine and lidocaine on reactive oxygen species (ROS) production by $(\mathbf{a}, \mathbf{b})$ unstimulated neutrophils and $(\mathbf{c}, \mathbf{d})$ after stimulation of the respiratory burst with phorbol myristate acetate (PMA) in the neutrophil cell subset with high-intensity fluorescence (rho+) in cord blood ( $n=12$; white bars) and adult control samples ( $n=7$; gray bars) after 1 and $4 \mathrm{~h}$ incubation. Intracellular ROS generation was assessed by flow cytometry using dihydrorhodamine 123 as a fluorescent probe. A subset of rho+ neutrophils was gated and analyzed separately. Data (median and interquartile range) are percentage of control values (without an anesthetic). ${ }^{*} P<0.05$, ${ }^{*} P<0.01, \S P<0.001$ compared with control (Friedman and Dunn). (a) In unstimulated rho+ neutrophil cell subset, no effect of bupivacaine on ROS production was observed. Differences between cord blood and adult control samples: $\uparrow P=0.031, \neq P=0.010$ (Mann-Whitney $U$-test). (b) Lidocaine increased ROS generation in unstimulated cord blood rho+ neutrophils at the lowest concentration applied (0.002 mmol/l). (c) Bupivacaine did not affect the PMA-elicited respiratory burst in cord blood rho+ neutrophils; in adult rho+ cells ROS suppression was detectable at lower concentrations applied. Differences between cord blood and adult neutrophils: $+P$ values for $0.0005,0.005$, and $0.05 \mathrm{mmol} / \mathrm{l}$ are $0.016,0.03$, and 0.047 , respectively $(1 \mathrm{~h}$ incubation); $\neq P$ values for 0.0005 , and $0.05 \mathrm{mmol} / \mathrm{l}$ are 0.010 , and 0.006 , respectively $(4 \mathrm{~h}$ incubation, Mann-Whitney $U$-test). (d) Lidocaine at $4 \mathrm{mmol} / \mathrm{l}$ decreased the PMA-induced respiratory burst in cord blood and adult blood rho+ neutrophil cell subsets after $1 \mathrm{~h}$. In cord blood, this effect did not persist after $4 \mathrm{~h}$. Differences between cord blood and adult rho + neutrophils: $\neq P$ values for $0.002,0.02,0.2$, and $4 \mathrm{mmol} / \mathrm{l}$ are $0.010,0.006,0.003$, and 0.047 , respectively ( $4 \mathrm{~h}$ incubation, Mann-Whitney $U$-test). 


\section{Articles $\mid$ Billertetal.}

Table 4. Summary of the major effects of bupivacaine and lidocaine on the ROS generation in cord blood and adult neutrophils

\begin{tabular}{|c|c|c|c|c|c|c|c|c|c|}
\hline & \multirow[b]{3}{*}{$\begin{array}{c}\mathrm{LA} \\
(\mathrm{mmol} / \mathrm{l})\end{array}$} & \multicolumn{4}{|c|}{ Entire sample } & \multicolumn{4}{|c|}{ rho+ subset } \\
\hline & & \multicolumn{2}{|c|}{ One hour incubation } & \multicolumn{2}{|c|}{ Four hours incubation } & \multicolumn{2}{|c|}{ One hour incubation } & \multicolumn{2}{|c|}{ Four hours incubation } \\
\hline & & Unstimulated & $\begin{array}{c}\text { PMA } \\
\text { stimulated }\end{array}$ & Unstimulated & $\begin{array}{c}\text { PMA } \\
\text { stimulated }\end{array}$ & Unstimulated & $\begin{array}{c}\text { PMA } \\
\text { stimulated }\end{array}$ & Unstimulated & $\begin{array}{c}\text { PMA } \\
\text { stimulated }\end{array}$ \\
\hline \multirow[t]{2}{*}{ Bupivacaine } & 0.0005 & & $C \downarrow, A \downarrow$ & & $\mathrm{A} \downarrow ; \mathrm{C}>\mathrm{A}$ & & $A \downarrow ; C>A$ & & $\mathrm{~A} \downarrow$ \\
\hline & 0.005 & & $\mathrm{~A} \downarrow$ & & & $C<A$ & $A \downarrow ; C>A$ & $\mathrm{C}<\mathrm{A}$ & $\mathrm{A} \downarrow$ \\
\hline \multirow[t]{4}{*}{ Lidocaine } & 0.002 & & & & $C>A$ & $C \uparrow$ & & & $C>A$ \\
\hline & 0.02 & & & & $\mathrm{~A} \downarrow$ & & & $\mathrm{A} \uparrow$ & $A \downarrow ; C>A$ \\
\hline & 0.2 & & & & $\mathrm{~A} \downarrow ; \mathrm{C}>\mathrm{A}$ & & & & $C>A$ \\
\hline & 4 & & $C \downarrow, A \downarrow$ & & $C \downarrow, A \downarrow$ & & $C \downarrow, A \downarrow$ & & $\mathrm{A} \downarrow ; \mathrm{C}>\mathrm{A}$ \\
\hline
\end{tabular}

Only significant differences are shown.

A, adult blood; C, cord blood; LA, local anesthetic; PMA, phorbol myristate acetate; $\downarrow$, decrease as compared to control; $\uparrow$, increase as compared to control.

at clinically relevant concentrations in neutrophils phagocytosing zymosan (24), however, in a whole-blood model, it had no significant effect at concentrations up to $10^{-4} \mathrm{M}$ on neutrophil priming and respiratory burst (12). The latter observations are in agreement with our findings. In the present study, the PMA-elicited respiratory burst was comparable between cord blood and adult neutrophils, which may be explained by similar protein kinase $\mathrm{C}$ membrane activity (25).

Across the entire neutrophil population, differences between cord blood and adult neutrophils regarding their response pattern to LAs were minor and could be detected only after longer exposure to the agents. Thus, we were not able to confirm our working hypothesis that LAs would suppress the respiratory burst to a significantly greater extent in cord blood neutrophils than in adult neutrophils. This hypothesis was based on the assumption that LAs impair the expression of cytochrome b558, a membrane compound of the NOX complex (26) that is dominant in fetal neutrophils at the cost of lower cytoplasmic component activity (15). Notably, suppression of cytoplasmic p47 phox translocation may underlie the inhibition of superoxide production in neutrophils exposed to chemical compounds, such as LAs (24).

After longer incubation, bupivacaine failed to have any effect on PMA-induced ROS generation in cord blood neutrophils. The transient suppression of the respiratory burst by bupivacaine suggests that it should not negatively affect this aspect of innate immunity in newborns. However, the longer incubation period in the whole-blood model may alter other factors, such as oxygen and glucose concentrations, which could influence the results (27).

Discrepancies between cord blood and adult neutrophils with regard to their response to LA exposure, albeit minor, should be viewed in light of the distinct functional characteristics of the fetal NOX complex (15). Other factors, like the stress associated with labor and blood acidemia, should also be considered. Both adrenergic stimulation and extracellular acidosis has been shown to interfere with $G_{\alpha q}$-dependent cellular activities and neutrophil functions $(28,29)$.
Neutrophils represent a highly heterogeneous cell population. The cytometric approach allows for cell subsets (different phenotypes and function) to be differentiated in various clinical and experimental settings (e.g., during labor and in both maternal and cord blood) $(30,31)$. In the present study, cells that showed high-intensity fluorescence (rho+) accounted for $5-7 \%$ of unstimulated neutrophils; this proportion corresponds to previous reports (32). Higher baseline ROS production in unstimulated rho+ neutrophils from cord blood, in line with other data, could reflect their higher responsiveness to upregulation of mediators of systemic inflammation, which is also a typical physiological feature of labor $(33,34)$. It could be supposed that circulating rho+ neutrophils represent the first line of defense, but their precise role remains unclear. Recent evidence suggests that, under conditions of systemic inflammation, circulating neutrophils exhibit "paradoxical functionality"; that is, selective priming of the respiratory burst coincides with a decrease in other aspects of antimicrobial potential (30). Nevertheless, our observation that bupivacaine failed to inhibit PMA-elicited ROS production in cord blood as opposed to the adult rho+ neutrophil subset indicates lower interference of bupivacaine in neutrophil function in the fetus and newborn than in adults.

The unexpected increase in ROS generation by unstimulated rho+ neutrophils following exposure to low concentrations of lidocaine could be viewed in light of its ability to induce mitochondrial derangement and possible secondary ROS induction (21). Interestingly, under clinical conditions, lidocaine was demonstrated to counteract the decrease in neutrophil respiratory burst during induction of anesthesia (34). Moreover, lidocaine is able to decrease cellular cAMP inversely associated with NOX activity via purinergic receptors and Goi proteins $(35,36)$.

The present study used an in vitro approach to evaluate the direct effect of LAs on fetal and neonatal host resistance potential. The data show that the inhibitory effect of bupivacaine on the neutrophil respiratory burst is short lived. Thus, in light of these findings, the use of bupivacaine in obstetric 
anesthesia seems to be justified. These findings may add to the broader discussion of the maternal and neonatal inflammatory consequences of neuraxial blockade for the relief of pain during labor. A recent meta-analysis supports previous reports regarding an increased risk for sepsis evaluation and antibiotic administration in neonates born to mothers who were administered epidural analgesia during labor (2). However, it should be stressed that during labor it is not only LAs administered by epidural infusion that could have an effect on fetal immune functions; a number of other factors, including analgesic adjuvants, the maternal stress response, fetal suffering, the duration of labor, drugs used to induce or augment labor, membrane rupture and microbial contamination, and antibiotics administered to the mother, could affect immune function.

\section{Study Limitations}

The present study does have some limitations. First, the wholeblood model, which more adequately mimics physiological conditions and avoids the use of cell separation procedures that could affect neutrophil ROS production, has some significant disadvantages. The results in this model could be influenced by interference from other cells and mediators. Because of plasma protein binding, it was impossible to set an exact desired concentration of the drugs being studied. In addition, the incubation time was restricted because of possible interference with sample hypoxia or glucose deprivation (27). Thus, observations made after $4 \mathrm{~h}$ incubation with LAs should be interpreted with caution.

Second, with regard to cord blood analysis, it should be noted that the study was performed under atmospheric oxygen tension; this setting may not reflect the natural in vivo hypoxic conditions of the fetus. Cord blood processing in a hypoxic microenvironment has been largely abandoned for technical reasons and may be associated with differences in cell performance (37).

Third, for technical reasons we restricted this study to two drugs. Extending the investigation to LA S-enantiomers used in obstetric anesthesia, namely ropivacaine and levobupivacaine, would be desirable in future studies.

\section{Conclusion}

In conclusion, using the whole-blood model, we have shown that LAs are capable of inhibiting the cord blood neutrophil respiratory burst for short-term. Further investigations in this field, both in vitro and in clinical settings, including evaluation of a broader range of LAs, is warranted.

\section{METHODS}

This study was approved by the Bioethics Commission (Poznan University of Medical Sciences). After mothers had provided written informed consent, umbilical blood was drawn immediately after cord clamping from 12 physiologically born healthy full-term neonates (six males and six females; 5-min Apgar score $\geq 9$ ). Peripheral venous blood was also obtained from seven control healthy, nonsmoking adults (males aged 20-40 y; median age 24 y). Blood was drawn into vacutainers with heparin (Sarstedt S-Monovette, Nuembrecht, Germany) and processed within $1 \mathrm{~h}$.

\section{Incubation of Whole Blood With LAs}

Stock solutions of $5 \mathrm{mmol} / \mathrm{l}$ bupivacaine (bupivacaine hydrochloride monohydrate; Sigma-Aldrich, St Louis, MO) and $20 \mathrm{mmol} / \mathrm{l} \mathrm{lido-}$ caine (lidocaine hydrochloride monohydrate; Sigma-Aldrich) were prepared in water and stored at $4{ }^{\circ} \mathrm{C}$. Whole blood specimens were incubated for 1 and $4 \mathrm{~h}$ under standard conditions $\left(37^{\circ} \mathrm{C} ; 5 \% \mathrm{CO}_{2}\right.$; HeraCell 150; ThermoScientific, Waltham, MA) with bupivacaine and lidocaine diluted in phosphate-buffered saline $(\mathrm{POCH}$, Gliwice, Poland) to obtain final concentrations in blood of 0.0005 (clinically relevant) (4), 0.005, 0.05, and $1 \mathrm{mmol} / \mathrm{l}$ bupivacaine and equipotent molar concentrations of $0.002,0.020,0.2$, and $4 \mathrm{mmol} / \mathrm{l}$ lidocaine.

\section{Flow Cytometric Measurement of Neutrophil ROS Generation}

After exposure to LA, some samples were incubated with $0.97 \mu \mathrm{mol} / \mathrm{l}$ PMA (Sigma-Aldrich) for $15 \mathrm{~min}$ at $37^{\circ} \mathrm{C}$. PMA is a universal extrareceptor cellular stimulator that activates protein kinase $\mathrm{C}$ signaling. Unstimulated and PMA-stimulated samples were analyzed separately. DHR 123 was used as the fluorescent probe. DHR 123 undergoes oxidative conversion to rhodamine, a two-electron oxidized fluorescent product, and is a measure of both intracellular cogenerated nitric oxide radical $(\bullet \mathrm{NO})$ and superoxide anion $\left(\bullet \mathrm{O}_{2}^{-}\right)(38)$. In the present study, $14.35 \mu \mathrm{mol} / \mathrm{l}$ DHR 123 (Sigma-Aldrich) was immediately after the incubation with PMA added to samples for $5 \mathrm{~min}$, at $37^{\circ} \mathrm{C}$. The reaction was stopped by placing the cells on ice. Erythrocytes were lysed with BD FACS Lysing Solution (BD FACS; Becton Dickinson, Franklin Lakes, NJ). After two washes with phosphate-buffered saline, cells were resuspended in phosphate-buffered saline and fluorescence was analyzed using a FACSCanto II Flow Cytometer (Becton Dickinson). Neutrophils were identified and gated based on their light scatter characteristics (side vs. forward scatter). At least 10,000 events were acquired for each analysis. A subset of neutrophils characterized by high-intensity fluorescence (rho+) was gated and analyzed separately. To identify rho+ neutrophils an electronic gate was set manually at the cut-off point of stimulated vs. unstimulated cells. Results were processed using Cell Quest Software (Becton Dickinson) and are expressed as MFI or as a percentage of control.

Data regarding the influence of LAs on the neutrophil ROS generation were presented separately in unstimulated and PMA-stimulated cells. Stimulation index was calculated only in the entire neutrophil population. This approach allowed us to gain a more detailed insight into the effects of the compounds in both unstimulated and stimulated cell state.

\section{Statistical Analysis}

Statistical analyses were performed using Statistica version 10 (StatSoft, Tulsa, OK) and GraphPad Prism 5 (GraphPad Software, San Diego, CA). Sample size was estimated separately for cord blood and adult control samples based on previous experiments and the presumption that lidocaine at the highest concentration used ( $4 \mathrm{mmol} / \mathrm{l})$ would cause a $50 \%$ decrease in ROS production. Assuming a power of 0.80 and $\alpha<0.05$, the calculated sample sizes for cord blood and adult control samples were 12 and 6, respectively.

Data were checked for normality by the Shapiro-Wilk test and are presented as the number, mean, and $95 \%$ confidence interval for normally distributed data, or as the median and interquartile range for non-normally distributed data. Because the laboratory data were not normally distributed, a non-parametric approach was used. The significance of differences between two groups was assessed by the Mann-Whitney $U$-test. Data involving more than two measurements were assessed using nonparametric Friedman's ANOVA with Dunn's multiple comparison tests for post hoc analysis. Two sided $P<0.05$ was considered significant.

\section{ACKNOWLEDGMENTS}

The authors are extremely grateful to Professors Maciej Kurpisz and Grzegorz Dworacki for their valuable comments and critical reading of the manuscript. The authors thank Stefania Burkowska, a coordinating midwife, for the help in collecting cord blood samples.

\section{STATEMENT OF FINANCIAL SUPPORT}

This project was supported by resources of Poznan University of Medical Sciences and, in part, by Polfa Warszawa SA.

Disclosure: The authors have no conflict of interest. 


\section{Articles $\mid$ Billertetal.}

\section{REFERENCES}

1. Agakidis C, Agakidou E, Philip Thomas S, Murthy P, John Lloyd D. Labor epidural analgesia is independent risk factor for neonatal pyrexia. J Matern Fetal Neonatal Med 2011;24:1128-32.

2. Heesen M, Klöhr S, Rossaint R, Straube S, Van de Velde M. Labour epidural analgesia and anti-infectious management of the neonate: a metaanalysis. J Perinat Med 2012;40:625-30.

3. Bedford Russell AR, Kumar R. Early onset neonatal sepsis: diagnostic dilemmas and practical management. Arch Dis Child Fetal Neonatal Ed 2015;100:F350-4.

4. Ala-Kokko TI, Alahuhta S, Arvela P, Vähäkangas K. Maternal, fetal and placental distribution of lidocaine-epinephrine and bupivacaine after epidural administration for cesarean section. Int J Obstet Anesth 1998;7:82-7.

5. Santos AC, Yun EM, Bobby PD, Noble G, Arthur GR, Finster M. The effects of bupivacaine, L-nitro-L-arginine-methyl ester, and phenylephrine on cardiovascular adaptations to asphyxia in the preterm fetal lamb. Anesth Analg 1997;85:1299-306.

6. Clark DA, Landaw SA. Bupivacaine alters red blood cell properties: a possible explanation for neonatal jaundice associated with maternal anesthesia. Pediatr Res 1985;19:341-3.

7. De Amici D, Gasparoni A, Chirico G, et al. Natural killer cell activity and delivery: possible influence of cortisol and anesthetic agents. A study on newborn cord blood. Biol Neonate 1999;76:348-54.

8. Gasparoni A, De Amici D, Ciardelli L, et al. Effect of lidocaine on neutrophil chemotaxis in newborn infants. J Clin Immunol 1998;18:210-3.

9. Birle A, Nebe CT, Hill S, Hartmann K, Poeschl J, Koch L. Neutrophil chemotaxis in cord blood of term and preterm neonates is reduced in preterm neonates and influenced by the mode of delivery and anaesthesia. PLoS One 2015; 10:e0120341.

10. Cassuto J, Sinclair R, Bonderovic M. Anti-inflammatory properties of local anesthetics and their present and potential clinical implications. Acta Anaesthesiol Scand 2006;50:265-82.

11. Ploppa A, Kiefer RT, Krueger WA, Unertl KE, Durieux ME. Local anesthetics time-dependently inhibit staphylococcus aureus phagocytosis, oxidative burst and CD11b expression by human neutrophils. Reg Anesth Pain Med 2008;33:297-303.

12. Ploppa A, Kiefer RT, Haverstick DM, Groves DS, Unertl KE, Durieux ME. Local anesthetic effects on human neutrophil priming and activation. Reg Anesth Pain Med 2010;35:45-50.

13. Melvan JN, Bagby GJ, Welsh DA, Nelson S, Zhang P. Neonatal sepsis and neutrophil insufficiencies. Int Rev Immunol 2010;29:315-48.

14. Komatsu H, Tsukimori K, Hata K, Satoh S, Nakano H. The characterization of superoxide production of human neonatal neutrophil. Early Hum Dev 2001;65:11-9.

15. Chudgar UH, Thurman GW, Ambruso DR. Oxidase activity in cord blood neutrophils: a balance between increased membrane associated cytochrome b558 and deficient cytosolic components. Pediatr Blood Cancer 2005;45:311-7.

16. Tsuchiya H, Ueno T, Mizogami M, Takakura K. Local anesthetics structure-dependently interact with anionic phospholipid membranes to modify the fluidity. Chem Biol Interact 2010;183:19-24.

17. Hattori M, Dohi S, Nozaki M, Niwa M, Shimonaka H. The inhibitory effects of local anesthetics on superoxide generation of neutrophils correlate with their partition coefficients. Anesth Analg 1997;84:405-12.

18. Mikawa K, Akamarsu H, Nishina K, Shiga M, Obara H, Niwa Y. Effects of ropivacaine on human neutrophil function: comparison with bupivacaine and lidocaine. Eur J Anaesthesiol 2003;20:104-10.
19. Welters ID, Menzebach A, Langefeld TW, Menzebach M, Hempelmann G. Inhibitory effects of S-(-) and R-(+) bupivacaine on neutrophil function. Acta Anaesthesiol Scand 2001;45:570-5

20. Kröller-Schön S, Steven S, Kossmann S, et al. Molecular mechanisms of the crosstalk between mitochondria and NADPH oxidase through reactive oxygen species-studies in white blood cells and in animal models. Antioxid Redox Signal 2014;20:247-66.

21. Kawasaki C, Kawasaki T, Ogata M, Sata T, Chaudry IH. Lidocaine enhances apoptosis and suppresses mitochondrial functions of human neutrophil in vitro. J Trauma 2010;68:401-8.

22. Mamiya K, Tomoda MK, Edashige K, Ueda W, Manabe M. Local anesthetics enhance nitric oxide production by human peripheral neutrophils. Physiol Chem Phys Med NMR 1995;27:111-9.

23. Hyvönen PM, Kowolik MJ. Dose-dependent suppression of the neutrophil respiratory burst by lidocaine. Acta Anaesthesiol Scand 1998;42:565-9.

24. Arakawa K, Takahashi H, Nakagawa S, Ogawa S. The effects of lidocaine on superoxide production and $\mathrm{p} 47 \mathrm{Phox}$ translocation in opsonized zymosanactivated neutrophils. Anesth Analg 2001;93:1501-6, table of contents.

25. Higuchi $H$, Nagahata $H$. Comparison of superoxide production, protein kinase $\mathrm{C}$ and tyrosine kinase activities in neutrophils from neonatal calves and cows. Res Vet Sci 1998;65:139-43.

26. Jinnouchi A, Aida Y, Nozoe K, Maeda K, Pabst MJ. Local anesthetics inhibit priming of neutrophils by lipopolysaccharide for enhanced release of superoxide: suppression of cytochrome b558 expression by disparate mechanisms. J Leukoc Biol 2005;78:1356-65.

27. Beitland S, Opdahl H, Aspelin T, Torjesen PA, Lyberg T. Reduction of glucose and insulin concentrations during in vitro incubation of human whole blood at different temperatures. J Diabet Res Clin Metabol 2013;2:11.

28. Sánchez-Fernández G, Cabezudo S, García-Hoz C, et al. G $\alpha q$ signalling: the new and the old. Cell Signal 2014;26:833-48.

29. Okajima F. Regulation of inflammation by extracellular acidification and proton-sensing GPCRs. Cell Signal 2013;25:2263-71.

30. Pillay J, Ramakers BP, Kamp VM, et al. Functional heterogeneity and differential priming of circulating neutrophils in human experimental endotoxemia. J Leukoc Biol 2010;88:211-20.

31. Ssemaganda A, Kindinger L, Bergin P, et al. Characterization of neutrophil subsets in healthy human pregnancies. PLoS One 2014;9:e85696.

32. Gessler P, Nebe T, Birle A, Haas N, Kachel W. Neutrophil respiratory burst in term and preterm neonates without signs of infection and in those with increased levels of C-reactive protein. Pediatr Res 1996;39:843-8.

33. Fredriksson MI. Effect of priming in subpopulations of peripheral neutrophils from patients with chronic periodontitis. J Periodontol 2012;83: 1192-9.

34. Swanton BJ, Iohom G, Wang JH, Redmond HP, Shorten GD. The effect of lidocaine on neutrophil respiratory burst during induction of general anaesthesia and tracheal intubation. Eur J Anaesthesiol 2001;18:524-9.

35. Benkwitz C, Garrison JC, Linden J, Durieux ME, Hollmann MW. Lidocaine enhances Galphai protein function. Anesthesiology 2003;99:1093-101.

36. Wang JP, Raung SL, Huang LJ, Kuo SC. Involvement of cyclic AMP generation in the inhibition of respiratory burst by 2-phenyl-4-quinolone (YT-1) in rat neutrophils. Biochem Pharmacol 1998;56:1505-14.

37. Roy S, Tripathy M, Mathur N, Jain A, Mukhopadhyay A. Hypoxia improves expansion potential of human cord blood-derived hematopoietic stem cells and marrow repopulation efficiency. Eur J Haematol 2012;88:396-405.

38. Kalyanaraman B, Darley-Usmar V, Davies KJ, et al. Measuring reactive oxygen and nitrogen species with fluorescent probes: challenges and limitations. Free Radic Biol Med 2012;52:1-6. 